\title{
Effects of expectations on loudness and loudness difference
}

\author{
Scott Parker • Julianne M. Moore • Sara Bahraini • \\ Kathleen Gunthert $\cdot$ Debra A. Zellner
}

Published online: 6 June 2012

(C) Psychonomic Society, Inc. 2012

\begin{abstract}
To determine how expectations affect loudness and loudness difference, in two experiments we induced some subjects to expect loud sounds (condition L), some to expect soft sounds (condition S), and others to have no particular expectations (control). In Experiment 1, all subjects estimated the loudnesses of the same set of three moderately loud $1-\mathrm{kHz}$ tones. Estimates were greatest for subjects in condition $\mathrm{S}$ and smallest for subjects in condition L. Control subjects' estimates were intermediate but closer to those of condition S subjects. In Experiment 2, subjects estimated the difference in loudness for pairs of moderately loud $1-\mathrm{kHz}$ tones. Again, estimates were smallest for condition L subjects; estimates were greatest for control subjects, and condition S subjects' estimates were closer to control estimates than to condition L estimates. This pattern of results is explainable by a combination of (1) Parducci's (1995) range-frequency theory and (2) a gain control mechanism in the auditory system under top-down governance (Schneider, Parker, \& Murphy, 2011).
\end{abstract}

Keywords Loudness $\cdot$ Expectation

Can expectations affect the perception of sensory intensity? For example, upon leaving your home when the outside

S. Parker $(\bowtie) \cdot J$. M. Moore $\cdot$ S. Bahraini $\cdot$ K. Gunthert Department of Psychology, American University,

Washington, DC 20016-8062, USA

e-mail: sparker@american.edu

D. A. Zellner

Department of Psychology, Montclair State University,

Montclair, NJ, USA temperature is 85 , does it feel hotter when the weather forecast said that today's high would be 75 than it would have if the forecast had said that the high would be 95 ? There has been little research into this sort of question.

Perhaps the most familiar domain in which expectancies have been studied is in illusions of heaviness - for example, the size-weight illusion and the material-weight illusion (see Ellis \& Lederman, 1998, for a history). In the sizeweight illusion, the larger of two objects with the same weight feels lighter. In the material-weight illusion, the one of two equally heavy objects that is made from what seems to be a heavier material seems lighter. The dominant theories of the illusions hold that expectations play a major role in the illusions (see, e.g., Buckingham \& Goodale, 2010; Buckingham, Ranger, \& Goodale, 2011; Ellis \& Lederman, 1998). Objects' weights are, to some degree, compared with their expected weights. The result is that deviations from expectations are magnified, and perceptions are contrasted away from their expected magnitudes. For example, Ellis and Lederman showed that whereas nongolfers (who had no expectations) judged what appeared to be practice and "real" golf balls of equal weight to be equally heavy, golfers (who expect practice balls to be much lighter than real golf balls) judged the apparent practice golf balls to be heavier than the real golf balls.

Another domain in which expectancies have been studied is time perception. The results of several studies (Boltz, 1989, 1993; Jones, Boltz, \& Klein, 1993) indicate that a given time interval is judged to have been shorter when it is shorter than expected than it is when it is longer than expected. So when there are expected durations, time intervals undergo contrast; perceptions move away from expectations. This sort of phenomenon appears to be a contributor to the return trip effect - the tendency for journeys to seem 
longer than the subsequent return trips (van de Ven, van Rijswijk, \& Roy, 2011).

The effect of expectations on taste intensity has also been studied. Olson and Dover (1979) found that ratings of coffee bitterness were lower for subjects who expected the coffee to be not bitter than for control subjects given no expectations. Caporale, Policastro, Carlucci, and Monteleone (2006) found that subjects' ratings of the bitterness and pungencies of various olive oils rose and fell with their expectations. Both of those studies found the same sort of assimilative result: Ratings migrated toward expectations. However Cardello and Sawyer (1992) found that expectations concerning the bitterness of pomegranate juice influenced subjects' ratings in a more complicated way, sometimes producing assimilation but sometimes producing contrast. Finally, Cardello, Melnick, and Rowan (1996) found that subjects' ratings of the taste intensity of a diluted orange drink exhibited contrast; subjects expecting low taste intensity rated the actual intensity higher than did subjects expecting high taste intensity.

So the answer to the question we posed at the beginning (will the forecast influence how the weather feels?) is "it well might." But the character of the influence is not readily predictable. The research on three perceptual domains exhibits varied results. The relationship of experience to expectation does not have a single pattern of consequences for perception of sensory intensity.

We here studied the effects of expectations in the loudness domain. We gave our subjects instructions that induced expectations of the loudnesses of the sounds they would hear.

Would a given sound's loudness be different if subjects expected a loud rather than a soft sound, and in what way?

There are several theoretical accounts suggesting that expectations should influence loudness judgments. One is Helson's (1964) adaptation-level (AL) theory. This theory says that stimuli are evaluated relative to some anchor value called the adaptation level. A stimulus will be perceived as more intense if it is above the AL than the same stimulus will be if it is below the AL. Thus, as Marks and Algom (1998) noted, "the model is geared to account readily for contrast effects" (p. 149). If expectations establish the value of AL, as Helson thought they might, one might predict that a given sound's loudness will vary with the subject's expectations, sounding softer when loud sounds are expected (i.e., when AL is higher) and louder when soft sounds are expected. Helson's theory is quite general and could apply to any perceptual domain.

A second theory that predicts contrast is Parducci's (e.g., 1965, 1974, 1995) range-frequency theory. An important aspect of this theory is that subjects tend to use the full range of responses available to them and try to distribute their responses more-or-less evenly over that range. Thus, when the stimulus range is expanded at the high end and truncated at the low end, stimuli within the range receive lower ratings than they do when the stimulus range is expanded at the low end and truncated at the high end. Similar results are obtained with skewing; if high stimulus values occur with greater frequency, stimuli in the middle of the distribution receive lower ratings. Therefore, a subject expecting loud sounds will give a moderate stimulus a low rating, wanting to reserve the upper end of the rating scale for the times that it's needed; the reverse will occur for a subject expecting soft sounds. Thus, a moderate sound will be rated lower by subjects expecting loud sounds than by subjects expecting soft sounds. Parducci (1974) allows for the possibility that such expectations might also alter perceptions as they alter ratings but does not definitely assert that they will.

A third theoretical account (Parker, Murphy, \& Schneider, 2002; Parker \& Schneider, 1994; Schneider \& Parker, 1990; Schneider, Parker, \& Murphy, 2011) is specifically about auditory intensity. It holds that the auditory system includes a nonlinear gain control mechanism that is under top-down cognitive control. A person can "turn down the gain" to protect against problematically loud sounds. Those authors found that the presentation of occasional unpredictable loud sounds within a series of softer sounds induced people to "turn down the gain." However, the presentation of occasional unpredictable soft sounds within a series of louder sounds did not induce people to "turn it up"; at least, any such effect was considerably weaker than the effect of the prospect of loud sounds. This model, then, predicts that a sound will seem softer for subjects expecting loud sounds than for subjects given no particular expectations but that a sound will seem, at most, only a little louder for subjects expecting soft sounds than for subjects in a noexpectation control condition.

In addition to their predictions about loudness judgments, these three theoretical approaches also make predictions about the effects of expectations on loudness difference judgments. AL theory (Helson, 1964; Peak, 1955) holds that stimuli remote from the AL attract particular attention (for evidence that this in fact occurs, see Escera, Corral, \& Yago, 2002). Attention to a particular range of auditory intensities is thought to enhance discrimination in that range (Nosofsky, 1983). Thus, on the assumption that expectation can establish the AL, Helson's theory predicts that sounds will seem very different if they are far from the expected level, because they will attract extra attention. This should occur irrespective of whether the sounds are louder than or softer than the expected level. So, for both subjects instructed to expect loud sounds and subjects instructed to expect soft sounds, loudness differences will seem larger than they do for subjects given no particular expectations. 
Parducci's (1995) range-frequency theory holds that stimulus range affects discrimination and, presumably, perceived difference. Two stimuli will be more discriminable and will, therefore, presumably appear more different when embedded in a narrow stimulus range than when in a broad range (a similar idea appears in Petzold, 1990; however, see Zellner et al., 2010, for data that violate that prediction). So if a subject expecting loud sounds or expecting soft sounds anticipates a stimulus range larger than does a subject given no particular expectations (e.g., because the subject expands a default "moderate" range in the direction of the expected sounds), that subject should rate differences as smaller than would a subject with no particular expectations. It is not certain, in our view, that loudness expectations would alter the expected range at all, let alone in this particular way. But were that to happen, subjects given those expectations should describe loudness differences as shrunken, according to range-frequency theory.

The gain control model makes a different prediction about loudness differences. The model says that when the gain is turned down, loudness differences and discriminability generally diminish. So, for example, Parker et al. (2002) studied subjects in an absolute loudness identification experiment with four "base" $1-\mathrm{kHz}$ tonal stimuli and investigated the consequence of adding a fifth stimulus remote in intensity from the "base" four. When the "base" stimuli were soft (all below $50 \mathrm{~dB}$ ) and the added fifth stimulus was $95 \mathrm{~dB}$, identification accuracy for the "base" stimuli was markedly reduced. (In addition, when the "base" stimuli were loud [all above $80 \mathrm{~dB}$ ] and the added fifth stimulus was $30 \mathrm{~dB}$, identification accuracy for the "base" four was also reduced, although considerably less so. Parker et al. speculatively attributed the latter reduction to some factor other than the operation of the gain control mechanism.) On the basis of those results, subjects instructed to expect loud sounds should find loudness differences to be much smaller than should subjects with no particular expectations; subjects instructed to expect soft sounds, however, should find loudness differences roughly equivalent to those for control subjects.

A singular feature of the gain control model is that the mechanism is presumed to be under top-down cognitive control, rather than simply being a passive responder to extremes of stimulation; thus, subjects' expectations should influence the setting of the amplifier. Expectations of loud sounds should induce subjects to turn down the gain, but expectations of soft sounds should have little or no effect. Thus, when loud sounds are expected and the gain is therefore reduced, two things should happen: (1) individual stimuli should sound softer, and (2) paired stimuli should seem less different. However, expectation of soft sounds should have no influence on judgments of loudness or loudness differences.
$\mathrm{AL}$ theory, range-frequency theory, and the gain control model differ, then, in their predictions about the effects of expectations on loudness and loudness difference. Both AL theory and range-frequency theory make predictions that are symmetric in that they make no distinction between the size of the effect produced by the expectation of loud versus soft sounds. The gain control mechanism, on the other hand, predicts an asymmetry of results: large consequences for loudness and loudness difference when loud sounds are expected and small or no consequences when soft sounds are expected.

We here report on two experiments in which we used instructions to manipulate subjects' expectations concerning the loudnesses of the stimuli they would hear. In Experiment 1, subjects rated the loudness of individual sounds. In Experiment 2 , subjects rated the difference in loudness between the two sounds in a pair. We will consider whether the results are consonant with any of the theoretical accounts: AL theory, range-frequency theory, and a topdown gain control mechanism in the auditory system.

\section{Experiment 1}

To investigate the effects of expectations on loudness, we compared the performances of subjects assigned to one of three conditions. The subjects in condition $\mathrm{L}$ were induced to expect loud sounds, the subjects in condition $\mathrm{S}$ were induced to expect soft sounds, and the subjects in a control condition were given no particular expectations concerning the loudnesses of the sounds they would hear. All subjects then rated the loudnesses of three tones. We compared the loudness ratings produced by the subjects in the three conditions. All subjects in all conditions heard and rated the same set of three stimuli.

\section{Method}

Subjects Sixty persons (chosen unsystematically from a student body approximately $60 \%$ female; apparent age range, 18-50 years) were recruited individually on the American University campus or at the bus stop serving the university's shuttle bus. Subjects were assigned to one of the three conditions and were run immediately upon recruitment at those outdoor locations. There were 20 subjects assigned to each of the three conditions. Recruitment and experimentation proceeded over several days.

Stimuli The stimuli were $500-\mathrm{ms} 1-\mathrm{kHz}$ tones with abrupt onsets and offsets created in Audacity and stored as .wav files in a Macintosh MacBook Pro computer (sampling rate was $44.1 \mathrm{kHz}$ ). They were delivered diotically over Sony 
MDR-NC 7 headphones (with the noise-cancellation off). The equipment used for calibration of sound levels included a GRAS KEMAR manikin (Model 45BA), Pinnae KB 0065 and 0066, a GRAS IEC 60711 Ear Simulator, a Bruel \& Kjaer 2250 sound level meter, an AO 0441 cable, a 4946 coupler, and preamplifier ZC 0032. The sound levels used in the experiment were 62.4, 72.0, and 78.5 dB SPL (hereafter, 62, 72 , and 79) - levels chosen to be moderately loud and mutually distinct. The amount of attenuation of environmental sound provided by the supraaural earphone cushions is unknown. Background noise levels were almost always below $65 \mathrm{~dB}$ A-weighted as measured by an Extech Model 407730 digital sound level meter. These levels of background noise should have had little consequence for the perceived loudnesses of the 1-kHz stimuli (Scharf, 1971).

Procedure Prospective subjects were approached and were asked whether they would be willing to participate in a brief psychology experiment. If they agreed, they were told that the study concerned people's experience of loudness, that they would hear several sounds and should rate them on a scale from 0 to 100, on which 0 meant silent and 100 meant very loud. The subjects were shown a visual representation of the scale - a horizontal line with its endpoints labeled. Subjects readily apprehend the use of a scale of this sort with no special instruction; this was particularly useful in the informal settings in which data collection occurred. Rating scales with a very large range give rise to numerical responses very similar to those produced by magnitude estimation (Foley, Cross, Foley, \& Reeder, 1983; Guirao, 1991; see also Marks \& Florentine, 2011). Noteworthily, judgments do not "jam up" at the high end of broad rating scales as they can with narrower rating scales (e.g., in Galanter \& Messick, 1961).

As the subject was taking the headphones and was about to put them on, the experimenter said one of three things, defining the three conditions of the experiment:

Condition L: "I hope these are not too loud."

Condition $S$ : "I hope these are not too soft."

Control: "I hope the instructions are clear."

All subjects in all conditions heard the set of tones in one of two sequences- $[62,79,72]$ or $[79,62,72]$. Stimulus sequence was completely crossed with condition. The subjects were assigned to the three conditions in blocks of 3 ; that is, of any 3 successive subjects, 1 was assigned to each of the three conditions.

\section{Results}

The mean ratings (and standard deviations) at each tone level for the subjects in each condition appear in the top three rows of Table 1 . Note that at all tone levels, ratings were lowest for the subjects in condition L ("I hope these are not too loud"), highest for the subjects in condition S ("I hope these are not too soft"), and intermediate for the subjects in the control condition ("I hope the instructions are clear").

Our interest was to see whether subjects in the three instructional conditions would rate the stimuli differently, and so our data analysis was targeted at that issue. At each of the three tone levels, we looked for those effects using a one-way ANOVA to compare the ratings in the three conditions. The results of those three ANOVAs appear in the fourth row of Table 1 . All three $F$-values were highly significant; the $p$-values are in the fifth row. The three estimates of the proportion-of-variance-accounted-for measure, $\omega^{2}$, appear in the sixth row of Table 1; these were large and roughly equal at approximately $75 \%$. At each of the three tone levels, we performed the three possible Tukey pairwise contrasts; at every tone level, all three conditions' ratings differed significantly from one another $(p<.002$ in all cases). (We reanalyzed the data using a Kruskal-Wallis test, which treats all responses as ordinal. All the conclusions above emerged from that analysis as well.)

We also wanted to see whether the influence of instructions was symmetrical - whether the two sets of expectationinducing instructions had equal and opposite effects on ratings, relative to the control instructions. If that were exactly true, the mean rating for the subjects in the control condition would be the average of the mean ratings for the subjects in the two expectation-inducing conditions (the increase in one perfectly balancing the decrease in the other). So, at each tone level, we performed a Scheffé contrast comparing the mean rating for control subjects with the average of the mean ratings for the subjects in the two expectation-inducing conditions. All three of those contrasts were significant $(p=.012$ for $62-\mathrm{dB}$ tones, $p=.003$ for both $72-$ and $79-\mathrm{dB}$ tones). At all three tone levels, the control subjects' mean was higher than the average of the means of the subjects in the two other conditions. This shows that condition $\mathrm{L}$ ratings were farther below control ratings than condition $\mathrm{S}$ ratings were above them. (This pattern was also confirmed in contrasts following the Kruskal-Wallis test.)

Table 1 Experiment 1: Mean ratings (and $S D$ s) for all stimuli in all conditions and ANOVA statistics for all stimuli

\begin{tabular}{llll}
\hline & 62-dB tone & 72-dB tone & 79-dB tone \\
\hline Condition L & $8.8(6.3)$ & $16.2(7.2)$ & $27.2(11.9)$ \\
Control & $36.4(9.1)$ & $50.4(9.6)$ & $64.2(9.2)$ \\
Condition $\mathrm{S}$ & $47.5(12.6)$ & $63.2(14.3)$ & $78.9(12.1)$ \\
$F(2,57)$ & 84.67 & 101.16 & 108.95 \\
$p$ & $<.00001$ & $<.00001$ & $<.00001$ \\
Estimated $\omega^{2}$ & .74 & .77 & .78 \\
\hline
\end{tabular}


Thus our instructional conditions had several big effects on loudness ratings. Relative to the ratings in the control condition, the instructions in condition $\mathrm{L}$ drove the loudness ratings down, whereas those in condition $\mathrm{S}$ drove the loudness ratings up. In addition, relative to the ratings in the control condition, condition $\mathrm{L}$ instructions lowered ratings more than condition $\mathrm{S}$ instructions raised them.

\section{Discussion}

The fact that subjects in the three conditions differed as they did is consistent with the predictions of Helson's (1964) AL theory. If expectations can establish the AL, the subjects will rate sounds relative to the AL. Thus, condition L subjects, who expected loud sounds (for whom the AL is therefore high), would rate the stimuli lower than would condition $\mathrm{S}$ subjects, who expected soft sounds (for whom the AL is therefore low), and control subjects should have an AL governed by their lifetime of experience with sound and, therefore, have both expectations and ratings intermediate between those of subjects in the other two conditions. That analysis accounts for the fact that the three conditions' mean ratings differed in just this way.

This feature of the data is also consistent with Parducci's (e.g., 1965) range-frequency theory. Condition L subjects, expecting loud sounds, should reserve the upper end of the rating scale for loud sounds and, therefore, give lower ratings to moderate stimuli than they would if they had no such expectations. Condition S subjects, expecting soft sounds, should reserve the lower end of the rating scale for those soft sounds and, therefore, give higher ratings to moderate stimuli than they would if they had no such expectations.

Both those theories predict the sort of contrast seen here. The top-down gain control model does not. In particular, it does not predict that subjects in condition S ("I hope these are not too soft") will differ from control subjects.

But a different feature of the data is predicted by the topdown gain control model, although not by either AL or range-frequency theory. That feature is the asymmetry between the departures from control data seen in condition $\mathrm{L}$ and condition $\mathrm{S}$ subjects. Neither AL nor range-frequency theory proposes that this sort of difference should occur. However, the top-down gain control model predicts only a small ratings increase, if any, for condition $\mathrm{S}$ subjects over control subjects but a large decrease for condition L subjects below control subjects. Our results are consistent with that prediction.

Our data suggest that two or three mechanisms are simultaneously at work in Experiment 1. Either or both of AL and range-frequency mechanisms will separate the responses of subjects in the three conditions. In addition, the top-down gain control mechanism will enhance the separation of condition L subjects from control subjects by inducing condition $\mathrm{L}$ subjects to turn down the gain in the auditory system. This additional separation of condition $\mathrm{L}$ subjects from control subjects, combined with either or both of the other two mechanisms, will result in the outcome seen in Experiment 1-clear differences between all conditions (provided by $\mathrm{AL}$ and/or range-frequency theory mechanisms) and an extra suppression in condition $L$ via the gain control. This is the first demonstration that the top-down gain control mechanism might affect loudness itself. Previous research on the model has investigated only loudness differences.

We pursued these ideas (AL theory, the range-frequency model, and top-down gain control) further in Experiment 2, in a setting where the three theories make distinct predictions.

\section{Experiment 2}

We now turn from ratings of the loudness of individual sounds to ratings of the difference in loudness between two sounds. To investigate the effects of expectations on loudness difference, we again instructed the subjects in condition L to expect loud sounds, the subjects in condition $\mathrm{S}$ to expect soft sounds, and the subjects in the control condition to have no particular expectations concerning the loudnesses of the sounds they would hear. All subjects then rated the loudness differences between the two tones in each of two tone pairs. We compared the magnitudes and patterns of loudness difference ratings produced by the subjects assigned to the three conditions. Recall that the three theories we are considering make somewhat different predictions about the experimental outcome. AL theory predicts that subjects told to expect either loud (condition L) or soft (condition S) sounds will find the actual moderate stimuli to differ more than will the no-expectation control subjects. Range-frequency theory predicts that subjects in both conditions $\mathrm{L}$ and $\mathrm{S}$ will find the actual moderate stimuli to differ less than will the no-expectation control subjects. The topdown gain control model predicts that subjects in condition $\mathrm{L}$ will find the actual moderate stimuli to differ less than will subjects in either condition $\mathrm{S}$ or the no-expectation control condition.

\section{Method}

Subjects Forty-eight persons (chosen unsystematically from a student body approximately $60 \%$ female; apparent age range, 18-50 years) were recruited individually on the American University campus or at the bus stop serving the 
university's shuttle bus. Immediately upon recruitment, subjects were assigned to one of the three conditions and were run at those outdoor locations, save for 2 who were recruited and run in the lobby of the Student Union building. There were 16 subjects assigned to each of the three conditions. (Three additional subjects erred when asked which of two sounds was the louder, and so we discarded their data and do not discuss it here.) Recruitment and experimentation proceeded over several days.

Stimuli The stimuli were pairs of $750-\mathrm{ms} 1-\mathrm{kHz}$ tones with abrupt onsets and offsets, with $750 \mathrm{~ms}$ between the tones. Stimuli were created in Audacity and stored as .wav files in a Macintosh MacBook Pro computer (sampling rate was $44.1 \mathrm{kHz}$ ). They were delivered diotically over Sony MDR-NC 7 headphones (with the noise-cancellation off). The process of stimulus calibration was that used in Experiment 1 . The stimulus intensities were 57.8 and $70.2 \mathrm{~dB}$ in pair 1 and 64.1 and 72.1 in pair 2, levels chosen to be moderately loud and mutually distinct. Background noise levels were, as in Experiment 1, almost always below $65 \mathrm{~dB}$ A-weighted. These levels of background noise should have had little consequence for loudness differences between the 1-kHz stimuli (Neff \& Jesteadt, 1996).

Procedure Prospective subjects were approached and were asked whether they would be willing to participate in a brief psychology experiment. If they agreed, they were told that the study concerned people's experience of loudness and that they would hear several pairs of sounds. They were then told that after each pair of sounds, they should indicate which sound (the first or the second) was the louder and they should then rate how much louder it had been on a scale from 1 to 10 . They were shown a visual representation of the scale they were to use-a horizontal line with 1 labeled slightly louder, 4 labeled somewhat louder, 7 labeled a lot louder, and 10 labeled very much louder. Subjects readily apprehend the use of a scale of this sort with no special instruction; this was particularly useful in the informal settings in which data collection occurred. This sort of 10-point scale has been used to assess hedonic differences in Zellner et al. (2010). As the subject was taking the headphones and was about to put them on, the experimenter said one of three things, defining the three conditions of the experiment:

Condition L: "I hope these are not too loud."

Condition S: "I hope these are not too soft."

Control: "I hope the instructions are clear."

The tone pairs could be presented in two sequences, and the tones within each pair might occur in either of two sequences; thus, there were eight possible stimulus sequences. Each sequence was used for 2 of the subjects in each condition, so sequence and condition were completely crossed. The subjects were assigned to the three conditions in blocks of 3; that is, of any 3 successive subjects, 1 was assigned to each of the three conditions.

\section{Results}

We averaged the difference ratings for the two tone pairs for each subject. The means (and standard deviations) of those averages for the subjects in the three conditions were the following: condition $\mathrm{L}, M=3.31(S D=0.56)$; condition $\mathrm{S}$, $M=5.66(S D=0.52)$; and control, $M=6.22(S D=0.79)$. Note that these average difference ratings were lowest for condition $\mathrm{L}$ subjects but that average difference ratings were similar for subjects in conditions $\mathrm{S}$ and control.

We investigated whether those average difference ratings were equivalent in the three conditions. The result of an ANOVA comparing them was $F(2,45)=88.72, p<$ .00001 , estimated $\omega^{2}=.79$. Tukey contrasts showed that ratings by subjects in condition $\mathrm{L}$ were significantly lower than ratings by both condition $\mathrm{S}$ and control subjects $(p<$ .00001 for both comparisons) and that ratings by condition $\mathrm{S}$ subjects were almost significantly lower than those by control subjects $(p=.051)$.

We wanted to determine whether condition L subjects' ratings were significantly farther below the control subjects' ratings than were condition $\mathrm{S}$ subjects' ratings. So we created the $99.9 \%$ confidence intervals for the two differences (control-condition L and control-condition S), using Tukey's approach. These two confidence intervals did not overlap. Thus, condition L subjects' difference ratings were significantly farther below the control subjects' ratings than were condition $\mathrm{S}$ subjects' ratings $(p<.001)$.

\section{Discussion}

The relationships among the loudness difference ratings for the three conditions do not conform to the predictions of $\mathrm{AL}$ theory (Helson, 1964). AL theory would predict that the difference ratings in both conditions $\mathrm{L}$ and $\mathrm{S}$ would be larger than those in the control condition. But the reverse was true: Conditions $\mathrm{L}$ and $\mathrm{S}$ both produced smaller difference ratings than did the control condition. Thus, the results of Experiment 2 are contrary to the predictions of AL theory.

Parducci's (1995) range-frequency theory makes predictions about the results of Experiment 2 if we presume that subjects in conditions $\mathrm{L}$ and $\mathrm{S}$ anticipated larger stimulus ranges than did control subjects. Under that assumption, range-frequency theory predicts that difference ratings will be smaller for subjects in conditions $\mathrm{L}$ and $\mathrm{S}$ than for control subjects. Difference ratings were indeed largest for 
control subjects; however, condition L subjects' difference ratings were markedly smaller than were condition $\mathrm{S}$ subjects'. This asymmetry is not predicted by range-frequency theory. In order for range-frequency theory to predict this asymmetry, it would need to assume that range expectations differed for subjects in conditions $\mathrm{L}$ and $\mathrm{S}$; in particular, range expectations for condition $\mathrm{L}$ subjects would have to be larger than range expectations for condition S subjects. We see no basis for that assumption. Thus, while rangefrequency theory does correctly predict that subjects in conditions $\mathrm{L}$ and $\mathrm{S}$ should give smaller difference ratings than will control subjects, it cannot account for the fact that condition $\mathrm{L}$ subjects gave smaller difference ratings than did condition $\mathrm{S}$ subjects.

However, this asymmetry between condition $\mathrm{L}$ and condition S subjects' difference ratings is predicted by the gain control model. That model predicts that condition L subjects should turn down the gain and, thereby, markedly reduce interstimulus difference. And indeed, condition L subjects gave by far the smallest ratings of loudness differences. The aspect of the results that the gain control model does not predict is that expectation of soft sounds also reduced difference ratings, a result consistent with range-frequency theory.

In all, AL theory fares badly in predicting the results of Experiment 2. But a combination of the gain control model and range-frequency theory does quite well. Rangefrequency theory can predict the reduction in difference ratings in both conditions $\mathrm{L}$ and $\mathrm{S}$. The gain control model predicts an enhanced reduction in difference ratings in condition L. This combination accounts for the totality of the results in Experiment 2.

\section{General discussion}

Three theoretical outlooks predicted aspects of the results of Experiment 1: Helson's (1964) AL theory, Parducci's (e.g., 1965) range-frequency theory, and the gain control model (e.g., Parker \& Schneider, 1994). For example, all of them predicted that subjects in condition $\mathrm{L}$ would give the lowest loudness ratings. However, AL theory failed badly in Experiment 2, predicting that subjects in conditions $\mathrm{L}$ and $\mathrm{S}$ would give the largest difference ratings, when in fact they gave smaller difference ratings than did subjects in the control condition. The results of Experiment 2 violate the predictions of AL theory, and we conclude that it does not contribute to the processes we studied.

Both the loudness contrast seen in Experiment 1 and the reduction of loudness differences seen in Experiment 2 are consistent with Parducci's (e.g., 1965, 1974) range-frequency theory if the "too loud" and "too soft" expectations are, in fact, expanding the cognitive range of expected stimuli in the direction of the expectation. However, the asymmetry seen in the degree of loudness contrast and the reduction of loudness differences suggests that there is also a gain control mechanism at work (e.g., Schneider et al., 2011). Expectation of loud sounds suppresses loudness and diminishes loudness difference more than does expectation of soft sounds. These results provide convergent evidence that the human auditory system includes a gain control mechanism under top-down control.

The auditory system has a variety of gain control mechanisms operating within it (Robinson \& McAlpine, 2009). One of them involves the system of efferents that go from the superior olive to the cochlea (Malmierca \& Ryugo, 2011; Schofield, 2011) via the olivocochlear bundles originating in the medial and lateral nuclei of the superior olivary complex. Those two connections serve to provide gain control in the cochlea and protect against acoustic trauma (Le Prell et al., 2003), one (the medial bundle) by effects on the outer hair cells and the other (the lateral bundle) by effects on afferents in the auditory nerve. The auditory cortex has efferent connections (sometimes with intermediaries) to both the medial and lateral nuclei of the olivary complex (Malmierca \& Ryugo, 2011; Mulders \& Robertson, 2000), so input to the auditory cortex might result in the cortex's activating this gain control system.

Two recent studies demonstrated that the responsiveness of the auditory cortex can be modulated by expectation. Todorovic, van Ede, Maris, and de Lange (2011) showed that in human subjects, repetition suppression (the reduction in magnetoencephalographically measured auditory cortex response to the second presentation of a stimulus) was greater for expected than for unexpected repetitions; in addition, they showed that response to an "omitted" tone was enhanced when subjects expected a repetition. Jaramillo and Zador (2011) showed that individual neurons in rat auditory cortex became progressively more responsive to task-irrelevant sounds as the expected moment of the critical target stimulus approached. Thus, stimulus-scheduleinduced expectations can modulate the auditory cortex's responses to acoustic stimuli. We think that our instructioninduced expectations can likewise alter the auditory cortex's responsiveness, which might, in turn, activate the descending gain control system.

The fact that our expectation induction altered loudness in Experiment 1 suggests that the gain control might be a contributor to the well-studied phenomenon called induced loudness reduction (ILR; see Epstein, 2007, for an extensive review). In ILR, a loud tone reduces the loudness of a subsequent sound at the same (or a near) frequency. For example, Wagner and Scharf (2006, Experiment 1) had subjects magnitude-estimate the loudnesses of $70-\mathrm{dB} 500-\mathrm{Hz}$ tones that were sometimes preceded by $80-\mathrm{dB} 500-\mathrm{Hz}$ inducer tones. Loudness estimates were lowered by the inducer tones. 
A phenomenon that bears some similarity to ILR is that of differential context effects (DCE; reviewed in Arieh \& Marks, 2011). In a typical experiment, subjects judge the loudness of sounds at two different frequencies with overlapping intensity ranges, sounds at one frequency being soft to moderate and those at the other being moderate to loud. Judgments of the stimuli in the overlap change dramatically depending on which frequency includes the loud sounds. Judgments are lowered for stimuli at the frequency that includes the loud sounds. Marks (1993) showed that the loud stimuli depress the loudness of others with the same frequency but leave sounds of the other frequency unaffected, a finding consistent with the operation of a band-limited gain control mechanism, as in Parker et al. (2002).

Arieh and Marks (2011) noted some points of dissimilarity between ILR and DCE in intensity tuning - the details of what particular louder intensities have how much impact on what particular lower-intensity stimuli. Nonetheless, Arieh and Marks refer to both processes as "adaptation-like." This description suggests that the reductions in loudness in ILR and DCE are reactions to the occurrence of the preceding loud stimuli.

However, the present experiments suggest an alternative view that is consistent with that of Parker et al. (2002). Experiment 4 of Parker et al. found that unpredictable loud sounds diminished the discriminability of soft sounds but that predictable loud sounds did not. They suggested that the threat of or expectation of a loud sound might originate from the experience of unpredictable loud sounds. That threat or expectation induced subjects to turn down their gain control mechanisms, reducing the loudness and discriminability of subsequent sounds. Here, we too show that the expectation of a loud sound reduces subjects' loudness ratings of subsequent sounds and their difference judgments. But in our studies, the gain control mechanism is called into operation via a simple expectation-inducing instruction: "I hope these are not too loud." The presentation of actual loud sounds is not necessary to produce the effect; the mere verbally communicated threat of loud tones suffices.

Therefore, we suggest that much if not all of the reduction in loudness seen in ILR and DCE might be due to the loud stimuli's inducing an expectation of additional intense stimuli. That expectation (rather than the previous loud sound itself) is what activates the top-down gain control mechanism, resulting in loudness reduction. Thus, in this way, ILR and DCE phenomena may be strategic and anticipatory, rather than merely reactive.

The asymmetry of effects in our Experiment 1 (greater impact on loudness estimates from expectation of loud sounds than of soft sounds) is consistent with the results of Parker et al. (2002) and Marks (1993, Experiment 15). It is also consistent with the fact that although induced loudness reduction occurs often, loudness enhancement as a separate phenomenon has been reported only rarely and with difficulty (see Arieh \& Marks, 2011, pp. 76-77). The asymmetry is not readily predicted by Parducci's range-frequency theory, irrespective of whether the theory is thought to apply only to a change in judgment strategy (1965) or also to the perceptions themselves (1974).

The visual system appears to have a top-down gain control system like the auditory system's, governing perception of luminance contrast rather than loudness (see de la Rosa, Gordon, \& Schneider, 2009; Mišić, Schneider, \& McIntosh, 2010). Paralleling the system in audition, it appears to function to protect the system from overload, in that high-contrast stimuli induce it to dampen contrast sensitivity for lower-contrast stimuli but low-contrast stimuli do not induce it to enhance contrast sensitivity for high-contrast stimuli. Whether it responds like the auditory system to verbal expectation-inducing instructions has not yet been studied. We know of no other sensory systems that have been shown to have such mechanisms. In particular, we do not know whether such mechanisms are involved in the perception of time, weight, or bitterness - three other sensory domains in which there is research on the influence of expectations on perceived intensity.

The experiments reported here give rise to rather clear conclusions. Expectations about loudness induce subjects to do two things. One is to adjust their strategy for rating loudnesses in line with Parducci's (1995) range-frequency theory; they do this both when soft sounds and when loud sounds are expected. The second is, when loud sounds are expected, to activate a protective gain control mechanism in the auditory system that diminishes both loudness and its rate of growth with auditory intensity.

Author Note We thank Matthew Bakke of Gallaudet University, Washington, DC, for the stimulus calibrations. We thank Bruce Schneider for helpful comments and advice on the manuscript. Julianne Moore was supported by a Mathias Undergraduate Summer Research Award from American University. Sara Bahraini is now at the Virginia Tech Carilion School of Medicine.

\section{References}

Arieh, Y., \& Marks, L. E. (2011). Measurement of loudness: Context effects. In M. Florentine, A. N. Popper, \& R. R. Fay (Eds.), Loudness (pp. 57-87). New York: Springer. doi:10.1007/978-14419-6712-1 3

Boltz, M. G. (1989). Time judgments of musical endings: Effects of expectancies on the "filled interval effect". Perception \& Psychophysics, 46, 409-418.

Boltz, M. G. (1993). Time estimation and expectancies. Memory \& Cognition, 21, 853-863.

Buckingham, G., \& Goodale, M. A. (2010). Lifting without seeing: The role of vision in perceiving and acting upon the size weight illusion. PLoS One, 5, e9709. doi:10.1371/journal.pone.0009709 
Buckingham, G., Ranger, N. S., \& Goodale, M. A. (2011). The material-weight illusion induced by expectations alone. Attention, Perception, \& Psychophysics, 73, 36-41. doi:10.3758/s13414010-0007-4

Caporale, G., Policastro, S., Carlucci, A., \& Monteleone, E. (2006). Consumer expectations for sensory properties in virgin olive oils. Food Quality and Preference, 17, 116-125. doi:10/1016/ j.foodqual.2005.07.011

Cardello, A. V., Melnick, S. M., \& Rowan, P. A. (1996). Expectations as a mediating variable in context effects. In I. Taub \& R. Bell (Eds.), Proceedings of the food preservation 2000 conference (Vol. 1, pp. 259-309). Hampton, VA: Science and Technology Corporation.

Cardello, A. V., \& Sawyer, F. M. (1992). Effects of disconfirmed consumer expectations on food acceptability. Journal of Sensory Studies, 7, 253-277.

de la Rosa, S., Gordon, M. S., \& Schneider, B. A. (2009). Knowledge alters visual contrast sensitivity. Attention, Perception, \& Psychophysics, 71, 451-462. doi:10.3758/APP.71.3.451

Ellis, R. R., \& Lederman, S. J. (1998). The golf-ball illusion: Evidence for top-down processing in weight perception. Perception, 27, 193-201.

Epstein, M. (2007). An introduction to induced loudness reduction. Journal of the Acoustical Society of America, 122, EL74-EL80. doi:10.1121/1.2761922

Escera, C., Corral, M.-J., \& Yago, E. (2002). An electrophysiological and behavioral investigation of involuntary attention towards auditory frequency, duration and intensity changes. Cognitive Brain Research, 14, 325-332.

Foley, H. J., Cross, D. V., Foley, M. A., \& Reeder, R. (1983). Stimulus range, number of categories, and the "virtual" exponent. Perception \& Psychophysics, 34, 505-512.

Galanter, E., \& Messick, S. (1961). The relation between category and magnitude scales of loudness. Psychological Review, 68, 363-372.

Guirao, M. (1991). A single scale based on ratio and partition estimates. In S. J. Bolanowski \& G. A. Gescheider (Eds.), Ratio scaling of psychological magnitude: In honor of the memory of S. S. Stevens (pp. 59-78). Hillsdale, NJ: Erlbaum.

Helson, H. (1964). Adaptation-level theory: An experimental and systematic approach to behavior. New York: Harper \& Row.

Jaramillo, S., \& Zador, A. M. (2011). The auditory cortex mediates the perceptual effects of acoustic temporal expectation. Nature Neuroscience, 14, 246-251. doi:10.1038/nn.2688

Jones, M. R., Boltz, M. G., \& Klein, J. M. (1993). Expected endings and judged duration. Memory \& Cognition, 21, 646-665.

Le Prell, C. G., Dolan, D. F., Schacht, J., Miller, J. M., Lomax, M. I., \& Altschuler, R. A. (2003). Pathways for protection from noise induced hearing loss. Noise \& Health, 5, 1-17.

Malmierca, M. S., \& Ryugo, D. (2011). Descending connections of auditory cortex to midbrain and brain stem. In J. A. Winer \& C. E. Schreiner (Eds.), The auditory cortex (pp. 189-208). New York: Springer. doi:10.1007/978-1-4419-0074-6 9

Marks, L. E. (1993). Contextual processing of multidimensional and unidimensional auditory stimuli. Journal of Experimental Psychology: Human Perception and Performance, 19, 227-249.

Marks, L. E., \& Algom, D. (1998). Psychophysical scaling. In M. H. Birnbaum (Ed.), Handbook of perception and cognition (2/e): Measurement, judgment and decision making (pp. 81-178). New York: Academic Press.

Marks, L. E., \& Florentine, M. (2011). Measurement of loudness: Part I. Methods, problems, and pitfalls. In M. Florentine, A. N. Popper, \& R. R. Fay (Eds.), Loudness (pp. 17-56). New York: Springer. doi:10.1007/978-1-4419-6712-1 2

Mišić, B. V., Schneider, B. A., \& McIntosh, A. R. (2010). Knowledgedriven contrast gain control is characterized by two distinct electrocortical markers. Frontiers in Human Neuroscience, 3, 1-12. doi:10.3389neuro.09.078.2009
Mulders, W. H. A. M., \& Robertson, D. (2000). Evidence for direct cortical innervation of medial olivocochlear neurones in rats. Hearing Research, 144, 65-72.

Neff, D. L., \& Jesteadt, W. (1996). Intensity discrimination in the presence of random-frequency multicomponent maskers and broadband noise. Journal of the Acoustical Society of America, 100, 2289-2298.

Nosofsky, R. (1983). Shifts of attention in the identification and discrimination of intensity. Perception \& Psychophysics, 33, 103112.

Olson, J. C., \& Dover, P. A. (1979). Disconfirmation of consumer expectations through product trial. Journal of Applied Psychology, 64, 179-189.

Parducci, A. (1965). Category judgment: A range-frequency model. Psychological Review, 72, 407-418.

Parducci, A. (1974). Contextual effects: A range-frequency analysis. In E. C. Carterette \& M. P. Friedman (Eds.), Handbook of perception Volume II: Psychophysical judgment and measurement (pp.127141). New York: Academic Press.

Parducci, A. (1995). Happiness, pleasure, and judgment: The contextual theory and its applications. Mahwah, NJ: Erlbaum.

Parker, S., Murphy, D., \& Schneider, B. (2002). Top-down gain control in the auditory system: Evidence from identification and discrimination experiments. Perception \& Psychophysics, 64, 598-615.

Parker, S., \& Schneider, B. (1994). The stimulus range effect: Evidence for top-down control of sensory intensity. Perception \& Psychophysics, 56, 1-11.

Peak, H. (1955). Attitude and motivation. In M. Jones (Ed.), Nebraska symposium on motivation: 1955 (pp. 149-189). Lincoln: University of Nebraska Press.

Petzold, P. (1990). The influence of anchor stimuli on judgments of attributes. In H.-G. Geissler, M. H. Muller, \& W. Prinz (Eds.), Psychophysical explorations of mental structures (pp. 138-146). Lewiston, NY: Hogrefe \& Huber.

Robinson, B. L., \& McAlpine, D. (2009). Gain control mechanisms in the auditory pathway. Current Opinion in Neurobiology, 19, 402407.

Scharf, B. (1971). Fundamentals of auditory masking. Audiology, 10, $30-40$.

Schneider, B. A., \& Parker, S. (1990). Does stimulus context affect loudness or only loudness judgments? Perception \& Psychophysics, 48, 409-418.

Schneider, B. A., Parker, S., \& Murphy, D. (2011). A model of top-down gain control in the auditory system. Attention, Perception, \& Psychophysics, 73, 1562-1578. doi:10.3758/ s13414-011-0097-7

Schofield, B. R. (2011). Central descending auditory pathways. In D. K. Ryugo, R. R. Fay, \& A. N. Popper (Eds.), Auditory and vestibular efferents (pp. 261-290). New York: Springer. doi:10.1007/978-1-4419-7070-1_9

Todorovic, A., van Ede, F., Maris, E., \& de Lange, F. P. (2011). Prior expectation mediates neural adaptation to repeated sounds in the auditory cortex: An MEG study. Journal of Neuroscience, 31, 9118-9123. doi:10.1523/JNEUROSCI.1425-11.2011

van de Ven, N., van Rijswijk, L., \& Roy, M. M. (2011). The return trip effect: Why the return trip often seems to take less time. Psychonomic Bulletin \& Review, 18, 827-832. doi:10.3758/s13423-0110150-5

Wagner, E., \& Scharf, B. (2006). Induced loudness reduction as a function of exposure time and signal frequency. Journal of the Acoustical Society of America, 119, 1012-1020. doi:10.1121/ 1.2159430

Zellner, D. A., Jones, K., Morino, J., Cogan, E. S., Jennings, E. M., \& Parker, S. (2010). Increased hedonic differences despite increases in hedonic range. Attention, Perception, \& Psychophysics, 72, 1261-1265. doi:10.3758/APP72.5.1261 